\title{
Quantitative Description of Pedestrian Dynamics with a Force-based Model
}

\author{
Mohcine Chraibi*, Armin Seyfried*, Andreas Schadschneider ${ }^{\dagger}$ and Wolfgang Mackens ${ }^{\ddagger}$ \\ ${ }^{*}$ Jülich Supercomputing Centre, \\ Forschungszentrum Jülich GmbH, 52425 Jülich, Germany \\ Email: m.chraibi@fz-juelich.de, a.seyfried@fz-juelich.de \\ $\dagger$ Institute of Theoretical Physics, University of Cologne, \\ D-50937 Cologne, Germany \\ Email: as@thp.uni-koeln.de \\ ${ }^{\ddagger}$ Hamburg University of Technology, \\ 21071 Hamburg, Germany \\ Email: mackens@tuhh.de
}

\begin{abstract}
This paper introduces a space-continuous forcebased model for simulating pedestrian dynamics. The main interest of this work is the quantitative description of pedestrian movement through a bottleneck. Measurements of flow and density will be presented and compared with empirical data. The results of the proposed model show a good agreement with empirical data. Furthermore, we emphasize the importance of volume exclusion in force-based models.
\end{abstract}

\section{INTRODUCTION}

Recently pedestrian dynamics has been gaining increasing interest. One focus of this research area is the security of people, e.g., trying to optimize evacuation processes by minimizing evacuation time. To achieve this "benchmark" architects and civil engineers need to have an idea about the minimal necessary width of exit doors, their optimal placement, length of escape routes, etc. Usually legal regulations and other descriptive specifications in handbooks provide requirements, which are in general not flexible enough for complex buildings. However, simulations of pedestrian dynamics offer the possibility to analyze and understand the dynamics of pedestrian streams in the building to be designed. This is useful to ease decisions e.g., dimensioning of emergency doors, and make them more realistic and adapted to different architectures.

In this work, we address the possibility of describing quantitatively the pedestrian dynamics, by proposing a spacecontinuous model. In section 2 the theory of mathematical modeling of pedestrian dynamics is briefly discussed. In section 3 our model is introduced. Then in section 4 numerical results of pedestrian flow through bottleneck and density measurements inside and in front of the entrance to a bottleneck will be presented. Section 5 gives concluding remarks.

\section{MATHEMAtiCAL MOdels}

There is a wide range of mathematical models to simulate pedestrian dynamics. Generally, these models are subdivided into macroscopic and microscopic models [7]. In macroscopic models the system is described by variables like crowd-density and flows of continua, whereas microscopic models consider the movement of individual persons separately. Microscopic models can be subdivided into models which are discrete or continuous in space. Widely-used discrete models for pedestrian dynamics are Cellular Automata models (CA), which describe phenomena in space-time by assigning discrete states to a grid of space-cells. These cells can be "occupied" by a pedestrian or "empty". Thus the movement of pedestrians in space is done by passing them from cell to cell in discrete time by simple rules that reflect e.g. psychological aspects of the motion.

Space-continuous force-based models determine the continuous movement of well distinguished individuals from their desires to reach certain targets and from influences of the space geometry as well as actual states and positions of the other individuals. Inspired by the field theory of Lewin [4], forcebased models assume that not only physical forces do affect the movement of pedestrians but similarly do social forces. A mathematical description of such social forces that determine the movement of pedestrians was first given by Helbing and Molnár in 1995 [2].

Though force-based models pose quite some computational problems that do not occur in $\mathrm{CA}$, their investigation appears to be worthwhile, since they permit higher resolution of geometry and time.

Force-based models take Newton's second law of dynamics as guiding principle. The movement of each pedestrian is thus described by

$$
\overrightarrow{F_{i}}=\sum_{i \neq j}^{\tilde{N}} \overrightarrow{F_{i j}^{r e p}}+\sum_{B} \overrightarrow{F_{i B}^{r e p}}+\overrightarrow{F_{i}^{d r v}}=m_{i} \overrightarrow{a_{i}}
$$

Here $\overrightarrow{F_{i j}^{r e p}}$ denotes the repulsive force exerted by pedestrian $j$ on pedestrian $i . \overrightarrow{F_{i B}^{r e p}}$ is the repulsive force emerging from borders (e.g. walls) and $\overrightarrow{F_{i}^{d r v}}$ is a driving force. $m_{i}$ is the mass of pedestrian $i$ and $\tilde{N}$ is the number of pedestrians acting on pedestrian $i$. Pedestrians try to avoid collisions and 


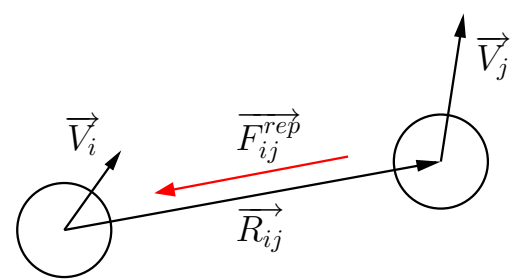

Fig. 1. Direction of the repulsive force acting on a pedestrian

contact with other pedestrians and objects by changing their direction. The repulsive forces are introduced to model this avoidance of physical contact. They can be social forces [2], [9], [12] as well as physical forces [1], [5], where physical forces come into play mainly to prevent overlapping with other pedestrians and with obstacles. Several different forms for these repulsive forces have been proposed. The driving force $\overrightarrow{F_{i}^{d r v}}$ represents the intention of a pedestrian to move towards a given destination.

The set of equations (1) for all pedestrians results in a high-dimensional system of second order ordinary differential equations. The time evolution of the positions and velocities of all pedestrians is then obtained by numerical integration.

Most force-based models describe the movement of pedestrians qualitatively well: Self-organisation phenomena e.g., lane formations [2], [1], [12], oscillations at bottlenecks [2], [1], "faster-is-slower" effect [3], [5], and clogging at exit doors [1], [12] are reproduced. These achievements indicate that the models could reliably describe pedestrian dynamics. However, a qualitative description is not sufficient if one has to make reliable statements about critical processes, e.g., emergency egress.

Moreover, rather often software implementations of the model do not rely on one sole approach. Especially in high density situations the simple numerical treatment has to be supplemented by additional techniques to obtain sensible results. Examples are restrictions on state variables and sometimes even totally different procedures which replace the above equations of motion (11) to avoid overlappings among pedestrians [3], [12] and negative and high velocities [2].

Necessarily a model has to describe pedestrian dynamics in a quantitative manner, e.g., by reproducing the fundamental diagram, the flow through bottlenecks and the density inside and in front of the entrance of a bottleneck. Additionally, for further investigation, it is advantageous that it uses a single set of forces.

In the next section, we propose such a model, which is based on a single set of formulae. Furthermore the model incorporates free parameters which allow adaption of the model to quantitative data.

\section{A Modified Centrifugal Force Model}

Pedestrians are modelled as circles moving in twodimensional space, see Figure 1. We model the repulsive force exerted by pedestrian $j$ on pedestrian $i$ as depending on the inverse of their distance. Additionally, relative velocity between two pedestrians is taken into account and assumed to influence the strength of the repulsive force. Moreover, we assume proportionality between the repulsive forces acting on a pedestrian $i$ and its desired velocity. Thus a pedestrian tending to move with high velocity "feels" intense repulsive actions.

The repulsive force, as a dimensionless relationship between the above mentioned quantities, is defined by

$$
\overrightarrow{F_{i j}^{r e p}}=-m_{i} K_{i j} \frac{\left(\nu\left\|\overrightarrow{V_{i}^{0}}\right\|+V_{i j}\right)^{2}}{\left\|\overrightarrow{R_{i j}}\right\|-\frac{1}{2}\left(D_{i}\left(\left\|\overrightarrow{V_{i}}\right\|\right)+D_{j}\left(\left\|\vec{V}_{j}\right\|\right)\right.} \overrightarrow{e_{i j}},
$$

where

$$
\overrightarrow{e_{i j}}=\frac{\overrightarrow{R_{i j}}}{\left\|\overrightarrow{R_{i j}}\right\|}, \quad \text { with } \quad \overrightarrow{R_{i j}}=\overrightarrow{R_{j}}-\overrightarrow{R_{i}},
$$

and

$$
V_{i j}=\frac{1}{2}\left[\left(\vec{V}_{i}-\vec{V}_{j}\right) \cdot \overrightarrow{e_{i j}}+\left|\left(\overrightarrow{V_{i}}-\overrightarrow{V_{j}}\right) \cdot \overrightarrow{e_{i j}}\right|\right],
$$

and

$$
K_{i j}=\frac{1}{2}\left[\frac{\overrightarrow{V_{i}} \cdot \overrightarrow{e_{i j}}+\left|\overrightarrow{V_{i}} \cdot \overrightarrow{e_{i j}}\right|}{\left\|\vec{V}_{i}\right\|}\right] .
$$

Here $\overrightarrow{V_{i}^{0}}$ denotes the desired velocity of pedestrian $i$ and $m_{i}$ its mas: $1 . V_{i j}$ is the projection of the relative velocity of pedestrian $j$ and pedestrian $i$ onto the direction of $\overrightarrow{e_{i j}},\left\|\overrightarrow{R_{i j}}\right\|$ is the distance between $i$ and $j, K_{i j}$ is a coefficient that reduces the action-field of the repulsive force to the angle of vision of each pedestrian $\left(180^{\circ}\right)$, and $\overrightarrow{e_{i j}}$ is the normalised directionvector between $i$ and $j .\|$.$\| denotes the Euclidian norm in$ $\mathbb{R}^{2}$ and $|$.$| the absolute value in \mathbb{R}$.

By means of the parameter $\nu$ the strength of the force can be adjusted while the diameter of pedestrians $D_{i}\left(\left\|\vec{V}_{i}\right\|\right)$ depends linearly on the velocity. This incorporates the dynamic space requirement of pedestrians, modeling the fact that faster pedestrians require more space than slower pedestrians, due to increasing step sizes [9].

$$
D_{i}=D_{a}+D_{b}\left\|\vec{V}_{i}\right\|
$$

with free parameters $D_{a}$ and $D_{b}$.

We use the driving force as defined in [2]:

$$
\overrightarrow{F_{i}^{d r v}}=m_{i} \frac{V_{i}^{0}-\left\|\overrightarrow{V_{i}}\right\|}{\tau} \overrightarrow{e_{i}^{0}},
$$

with $\overrightarrow{V_{i}}$ the velocity of pedestrian $i, \overrightarrow{e_{i}^{0}}$ its desired direction and $\tau$ a time constant.

The above model has been developed from the Centrifugal Force Model (CFM) of Yu et al. [12]. Their expression for the repulsive force reads as follows:

$$
\overrightarrow{F_{i j}^{r e p}}=-m_{i} K_{i j} \frac{V_{i j}^{2}}{\left\|\overrightarrow{R_{i j}}\right\|} \overrightarrow{e_{i j}},
$$

\footnotetext{
${ }^{1}$ In all simulations the mass is set to one for all pedestrians.
} 
with the quantities $K_{i j}, V_{i j}, \overrightarrow{R_{i j}}$ and $\overrightarrow{e_{i j}}$ as defined in Equations (5), (4), and (3).

When pedestrians come near to one another the repulsive force is expected to grow. In the CFM, this is in general not the case due to the relative velocity term in the nominator of the repulsive force (8). If both the distances between two pedestrians and their relative velocities are small, their quotient does not grow as expected. Consequently the repulsive force does not become high enough to prohibit overlappings. Introducing the intended speed in the nominator of the repulsive force (2) we eliminate this side-effect. Furthermore, there is a compensation between repulsive and driving forces at low velocities, which damps oscillations. Due to these changes we can do in most cases without the extra Collision Detection Technique (CDT) which takes over control in [12] in case of formation of dense crowds.

\section{NUMERICAL RESULTS}

The solution of the initial value problem (1) was done in all simulations using a fourth-order Runge-Kutta scheme with a fixed-step size of $\Delta t=0.01 \mathrm{~s}$. The repulsive interaction between two pedestrians is neglected when the distance between them is larger than two meters.

In order to investigate the influence of our modifications, different model approaches were simulated. Then the results were compared with empirical data of pedestrian flow through a bottleneck.

The flow of 60 pedestrians through the bottleneck situation as described in [8] was simulated. The width of the bottleneck was changed from $0.8 \mathrm{~m}$ to $1.2 \mathrm{~m}$ in steps of $0.1 \mathrm{~m}$. The free parameter $\nu$ in Equation (2) is set to $0.28 \mathrm{~m} / \mathrm{s}$ for the repulsive force among pedestrians and to $0.4 \mathrm{~m} / \mathrm{s}$ for the repulsive force emerging from obstacles. For the free parameters in Equation (6) we set

$$
D_{a}=0.2 \mathrm{~m} \text { and } D_{b}=0.2 \mathrm{~s} \text {. }
$$

Several parameter values were tested. With this parameter set the results of the simulations are in good agreement with the empirical flow measurements presented in [7], see Figure 2(a) For comparison the flow through the same bottleneck was measured by the original CFM without CDT. The results presented in Figure 2(b) show very high values of the flow. These are possible, because CDT does no more care for volume exclusion.

Actually, control of bottleneck-flow seems to be dominated by the CDT in the CFM-approach. To back this hypothesis we measured pedestrian flow through the bottleneck with a CFM like model without repulsive forces, managing collisions with CTD, only. The gained values are in the range of experimental data, see Figure 2(c) Volume exclusion of pedestrians seems thus to be a favorable feature of successful models.

A second validation of our modifications of CFM comes from measurements of density inside the bottleneck as well as in front of the entrance to the bottleneck, see Figure 3 . The density in front of the entrance to the bottleneck is presented in Figure 4(a). The results are in good agreement

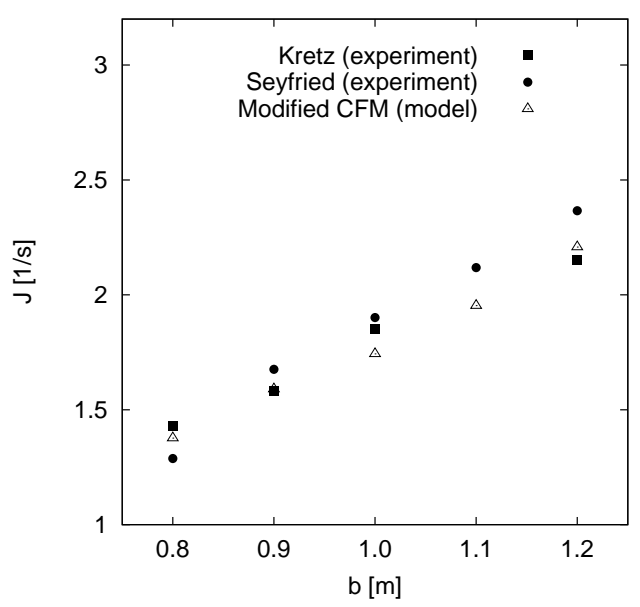

(a) Flow measurement with the modified CFM without CDT

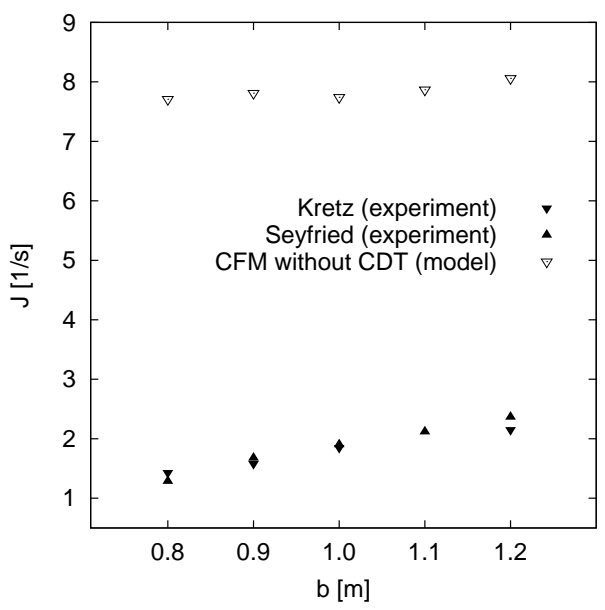

(b) Flow measurement with original CFM without CDT. Without CDT the flow values are unrealisticly high.

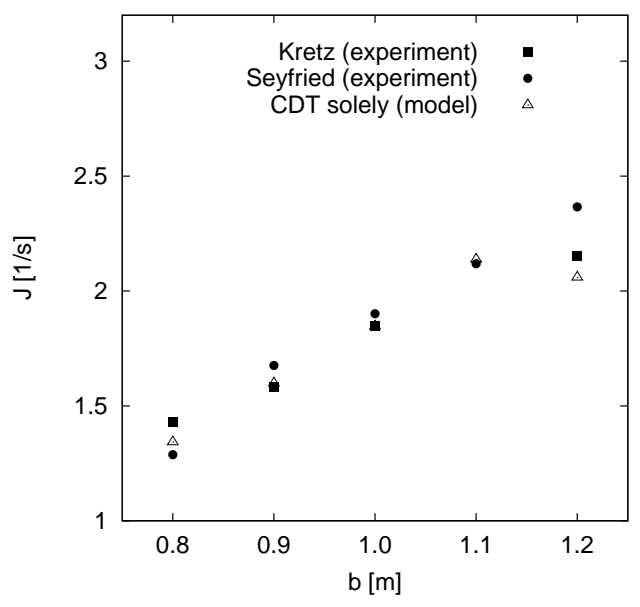

(c) Flow measurement with CDT without repulsive forces.

Fig. 2. Flow measurement with tree different models in comparison with empirical data [7]. 
with the experimental data in [10]. Additionally, the measured density values inside the bottleneck are in accordance with the published empirical results in [8], see Figure 4(b)

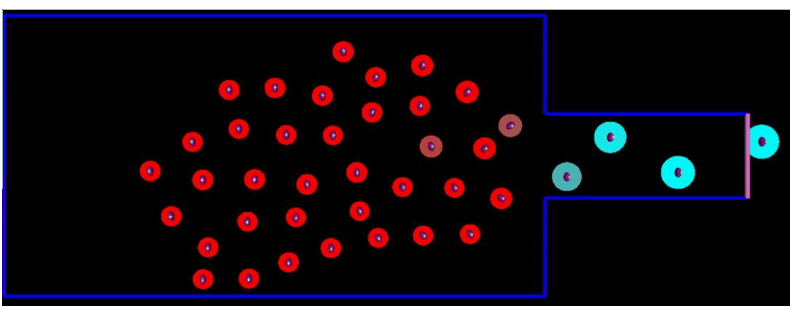

Fig. 3. Screenshot of a simulation: The density of pedestrians is measured by counting the number of pedestrians that are located inside one of the measurement rectangles at each integration time. The area of the appropriate rectangle divides the result.

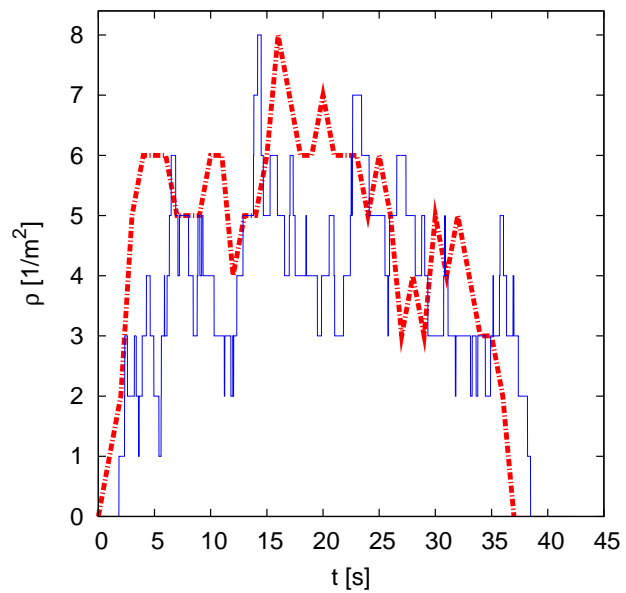

(a) Density in front of the entrance to the bottleneck

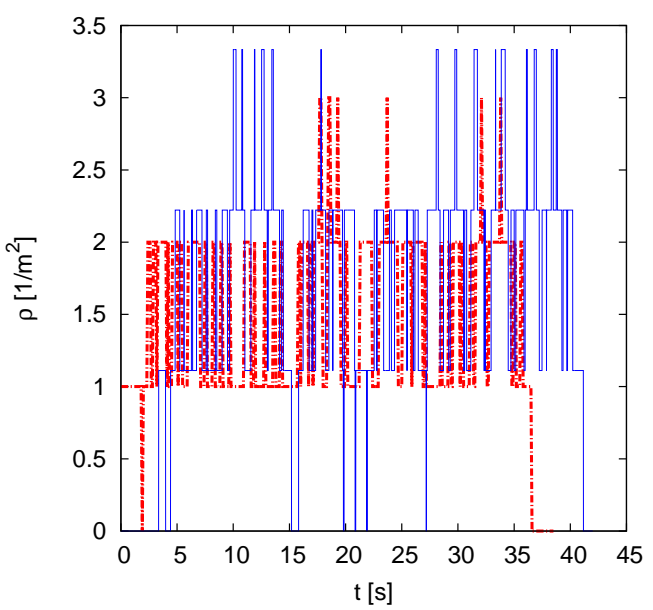

(b) Density inside the bottleneck

Fig. 4. Density measurements: The simulation results (blue lines) are in good agreement with the empirical data presented resp. in [10] and [8].

Though the model is already a useful tool to describe quantitatively the bottleneck-situations as defined in [8], it is not yet fully satisfactory. Depending on the specific situation oscillations and overlappings can still occur. Determination of uniformly usable parameters is a difficult endeavour; cf. the parameter estimation in SimWalk [11].

\section{CONClusion}

The proposed space-continuous force-based model quantitatively describes the movement of pedestrians in 2D-space in an almost satisfactory way. Besides being a remedy for numerical instabilities in CFM the model simplifies the approach of $\mathrm{Yu}$ et al. since we can dispense with their extra CDT without deteriorating performance. The implementation of the model is straightforward and does not use any restrictions on the velocity as was the case e.g., in [2].

Simulation results show good agreement with empirical data. The model contains natural free parameters which can further be tuned to adapt the model to certain scenarios. Introducing density-dependence of the strength of the repulsive force promises further ameliorations of the model.

Furthermore we have provided some evidence that volume exclusion is an important issue in describing pedestrian dynamics.

\section{REFERENCES}

[1] D. Helbing. Collective phenomena and states in traffic and self-driven many-particle systems. Computational Materials Sciencs, 30:180-187, 2004.

[2] D. Helbing and P. Molnár. Social force model for pedestrian dynamics. Phys. Rev. E, 51:4282-4286, 1995.

[3] T. I. Lakoba, D. J. Kaup, and N. M. Finkelstein. Modifications of the Helbing-Molnár-Farkas-Vicsek Social Force Model for Pedestrian Evolution. Simulation, 81:339-352, 2005.

[4] Kurt Lewin, editor. Field Theory in Social Science. Greenwood Press, Publishers, 1951.

[5] D. R. Parisi and C. O. Dorso. Morphological and dynamical aspects of the room evacuation process. Physica A: Statistical Mechanics and its Applications, 385(1):343-355, 2007.

[6] C. Rogsch, A. Seyfried, and W. Klingsch. Comparative Investigations of the Dynamical Simulation of Foot Traffic Flow. In Pedestrian and Evacuation Dynamics 2005, pages 357-362. Springer, 2006.

[7] A. Schadschneider, W. Klingsch, H. Kluepfel, T. Kretz, C. Rogsch, and A. Seyfried. Evacuation Dynamics: Empirical Results, Modeling and Applications. Encyclopedia of Complexity and System Science. Springer, 2009.

[8] A. Seyfried, T. Rupprecht, O. Passon, B. Steffen, W. Klingsch, and M. Boltes. New insights into pedestrian flow through bottlenecks. Transportation Science, in print, 2009. http://arxiv.org/abs/physics/0702004

[9] A. Seyfried, B. Steffen, and T. Lippert. Basics of modelling the pedestrian flow. Physica A, 368:232-238, 2006.

[10] A. Seyfried, B. Steffen, T. Winkens, A. Rupprecht, M. Boltes, and W. Klingsch. Empirical data for pedestrian flow through bottlenecks. In Traffic and Granular Flow 2007. Springer, in print, 2009.

[11] Albert Steiner, Michel Philipp, and Alex Schmid. Parameter Estimation for a Pedestrian Simulation Model. In Swiss Transport Research Conference, 2007

[12] W. J. Yu, L.Y. Chen, R. Dong, and S.Q. Dai. Centrifugal force model for pedestrian dynamics. Phys. Rev. E, 72(2):026112, 2005. 